\title{
The modulatory effect of electrical stimulation on the excitability of the corticospinal tract varies according to the type of muscle contraction being performed
}

\section{Kei Saito ${ }^{1,2}$ *, Kenichi Sugawara ${ }^{3}$, Shota Miyaguchi $^{2}$, Takuya Matsumoto $^{2}$, Hikari Kirimoto², Hiroyuki Tamaki ${ }^{1,2}$ and Hideaki Onishi ${ }^{1,2}$}

\author{
${ }^{1}$ Department of Physical Therapy, Niigata University of Health and Welfare, Niigata, Japan \\ 2 Institute for Human Movement and Medical Sciences, Niigata University of Health and Welfare, Niigata, Japan \\ ${ }^{3}$ Faculty of Rehabilitation, Kanagawa University of Human Services, Kanagawa, Japan
}

\section{Edited by: \\ Rachael D. Seidler, University of \\ Michigan, USA}

Reviewed by:

Sean Kevin Meehan, University of Michigan, USA

Kevin Power, Memorial University, Canada

\section{*Correspondence:}

Kei Saito, Department of Physical Therapy, Niigata University of Health and Welfare, 1398 Shimami-cho, Kita-ku, Niigata 950-3198, Japan e-mail: kei-saito@nuhw.ac.jp

\begin{abstract}
Afferent input caused by electrical stimulation of a peripheral nerve increases corticospinal excitability during voluntary contractions, indicating that proprioceptive sensory input arriving at the cortex plays a fundamental role in modulating corticospinal excitability. The purpose of this study was to investigate whether the effect of electrical stimulation on the corticospinal excitability varies according to the type of muscle contraction being performed. Motor-evoked potentials (MEPs) were elicited by transcranial magnetic stimulation (TMS) during a shortening contraction, an isometric contraction, or no contraction of the first dorsal interosseous (FDI) muscle. In some trials, electrical stimulation of the ulnar nerve was performed at $110 \%$ of the sensory threshold or $110 \%$ of the motor threshold prior to TMS. Electrical stimulation involved either a train of 50 pulses at $10 \mathrm{~Hz}$ or a single pulse. Shortening contraction with the train of electrical stimuli significantly increased MEP amplitudes, and the increase was dependent on the type of stimulation. Isometric contraction with the train of electrical stimuli and electrical stimulation without voluntary contraction did not affect MEP amplitudes. A single pulse of electrical stimulation did not affect MEP amplitudes in any condition. Thus, electrical-stimulation-induced modulation of corticospinal excitability varied according to the type of muscle contraction performed and the type of stimulation. These results show that the type of contraction should be considered when using electrical stimulation for rehabilitation in patients with central nervous system lesions.
\end{abstract}

Keywords: electrical stimulation, voluntary contraction, excitability, corticospinal tract, transcranial magnetic stimulation

\section{INTRODUCTION}

Application of electrical stimulation concurrent with voluntary movement facilitates motor recovery (Bolton et al., 2004; de Kroon etal., 2005; McDonnell etal., 2007; Hara etal., 2013) and induces substantial cortical reorganization in patients with central nervous system injuries such as stroke (Kimberley et al., 2004; Bhatt et al., 2007; Fujiwara et al., 2009; Hara et al., 2013). A neuroimaging study using transcranial magnetic stimulation (TMS) indicated that electrical stimulation with voluntary movement resulted in increased corticospinal excitability concomitantly with functional motor recovery in stroke patients (Tarkka etal., 2011). Thus, increasing corticospinal excitability is considered to be involved in the mechanism underlying functional motor recovery from stroke using this combined therapeutic approach.

The stimulation intensity was effective in modulating the change in corticospinal excitability by electrical stimulation with voluntary movement. When the stimulated muscle is voluntarily shortened, low-intensity electrical stimulation displays significantly increased corticospinal excitability than high-intensity electrical stimulation (Saito et al., 2013). However, the effect of stimulation intensity on corticospinal excitability is considered to depend on whether or not the stimulated muscle is active. Chipchase et al. (2011b) reported that the stimulation intensity is strongly associated with the modulation of corticospinal excitability when electrical stimulation is delivered without voluntary movement. Khaslavskaia et al. (2002) showed that electrical stimulation increases the excitability of the corticospinal projection to the stimulated muscle in an intensity-dependent manner when the stimulated muscle is quiescent.

Voluntary movement differs in its ability to modulate the corticospinal excitability in response to the type of muscle contraction being performed. A previous study in monkeys showed that somatosensory input evoked by voluntary movement is quantitatively different between the types of muscle contraction (Seki and Fetz, 2012). Lee and White (1974) reported that shortening contractions increased the somatosensory evoked potential (SEP) greater than did isometric contractions. These studies indicate that somatosensory input by shortening contraction is quantitatively larger than input by isometric contraction. Together with the observation that shortening contractions increased corticospinal excitability significantly more than did isometric contraction 
(Chye et al., 2010), movement related sensory gating is considered to be associated with modulation of corticospinal excitability.

The synergistic effect of afferent input by electrical stimulation and somatosensory input by voluntary movement is known to play an important role in modulating corticospinal excitability (Kido Thompson and Stein, 2004; Khaslavskaia and Sinkjaer, 2005; Barsi et al., 2008; Saito et al., 2013; Sugawara et al., 2013). However, it remains unknown whether the effect of stimulation intensity on corticospinal excitability is dependent on the type of muscle contraction being performed. The somatosensory and electrical afferent inputs both differ with the type of muscle contraction and stimulation intensity; thus, it is important to clarify the optimal stimulation intensity to effectively increase corticospinal excitability for a given type of muscle contraction combined with electrical stimulation. Thus, we examined the influence of stimulation intensity on the change in corticospinal excitability by electrical stimulation in response to the type of voluntary movement. The results of this study may indicate the appropriate intensity of electrical stimulation for treatment of motor dysfunction using voluntary movement concurrent with electrical stimulation.

The purpose of this study was to elucidate the effect of electrical stimulation of the ulnar nerve on the excitability of the corticospinal projection to the first dorsal interosseous (FDI) muscle during voluntary shortening contractions and voluntary isometric contractions.

\section{MATERIALS AND METHODS PARTICIPANTS}

Fourteen neurologically normal right-handed volunteers (thirteen males and one female) with a mean age of $22.1 \pm 1.7$ years participated in this study. All volunteers provided written informed consent before participation. This study was performed in accordance with the Declaration of Helsinki, and the protocol was approved by the Research Ethics Committee of Niigata University of Health and Welfare.

\section{ELECTROMYOGRAM RECORDING}

During all experiments, the participant sat comfortably in a chair and placed his or her right hand on a table with the palm perpendicular to the horizontal plane. Surface electromyograms were recorded from the right FDI muscle using disposable silver-silver chloride surface electrodes (N-00-S; Mets Inc., Tokyo, Japan).

\section{ELECTRICAL STIMULATION OF THE ULNAR NERVE}

Electrical nerve stimulation was applied to the right wrist to stimulate the ulnar nerve that innervates the FDI muscle. Stimulation was generated using an electrical generator (SEN-7203; Nihon Kohden Co., Tokyo, Japan) with an isolator (SS-104; Nihon Kohden Co.) and a pair of silver-silver chloride surface electrodes. Stimulation was delivered in trains of 50 pulses at $10 \mathrm{~Hz}$ with a pulse width of $1 \mathrm{~ms}$. Two different stimulus intensities were used: (i) $110 \%$ of the motor threshold (above motor threshold) and (ii) $110 \%$ of the sensory threshold (above sensory threshold). The motor threshold was defined as the lowest stimulus that evoked a visible twitching of the index finger, and the sensory threshold was defined as the lowest stimulus that the volunteer could perceive. In this study, the average motor threshold was $11.2 \pm 4.7 \mathrm{~mA}$ (mean $\pm \mathrm{SD}$ ), and the average sensory threshold was $7.4 \pm 2.7 \mathrm{~mA}$.

\section{VOLUNTARY HAND MOVEMENT TASKS}

The volunteers were asked to perform the following hand movement tasks: (i) shortening contraction and (ii) isometric contraction (Figure 1) to investigate how the influence of electrical stimulation on the excitability of the corticospinal tract was altered by the difference in somatosensory input induced by voluntary hand movement. In the shortening contraction task, the volunteer flexed the metacarpophalangeal (MP) joint of the index finger from 0 to $90^{\circ}$ while the index finger received external torque from a custom-made apparatus that connected distal interphalangeal (DIP) joint of the index finger to a scale weight via a pulley to easily sustain constant activity of FDI muscle (Figure 1). The shortening contraction was a single movement which was held for $5 \mathrm{~s}$. In the isometric contraction task, the volunteer performed an isometric pinch movement, attempting to touch the index finger to the thumb. The index finger was passively separated from the thumb by the same custom-made apparatus (Figure 1), and the volunteer was required to maintain the MP joint of the index finger at $90^{\circ}$ for the 5-sec duration of the task. In both hand movements, the volunteer set the EMG of the FDI muscle to 15\% MVC on the basis of visual EMG feedback during voluntary hand movement; this procedure was practiced until reaching an EMG of $15 \%$ MVC.

\section{TMS}

Transcranial magnetic stimulation was delivered over the left primary motor cortex using a figure-eight coil with an internal wing diameter of $90 \mathrm{~mm}$ connected to a Magstim 200 (Magstim Co. Ltd., Whitland, UK). The optimal site for eliciting motor-evoked potentials (MEPs) from the FDI (motor hotspot) was found by delivering a slightly suprathreshold stimulus at $1-\mathrm{cm}$ intervals around the assumed motor hotspot. The coil was placed tangentially to the scalp and held at $45^{\circ}$ to the midsagittal line. The TMS intensity was set to $120 \%$ of the resting motor threshold for tasks that involved no voluntary contraction and $120 \%$ of the active motor threshold for

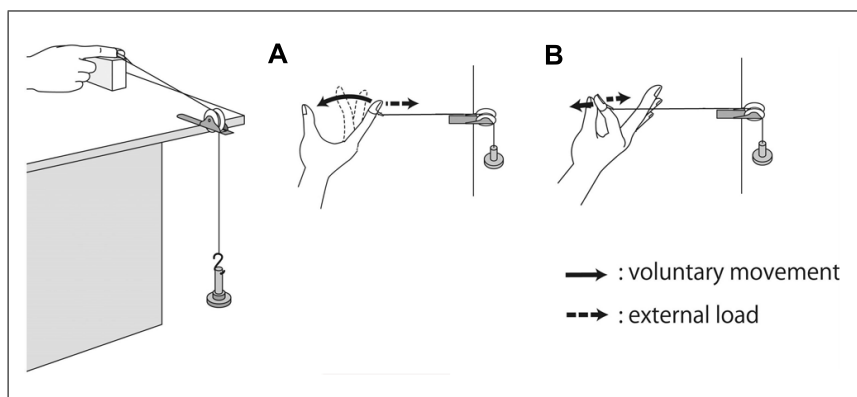

FIGURE 1 | Experimental set-up and voluntary hand movement. (A) For the shortening contraction task, the participant was required to flex the metacarpophalangeal (MP) joint of the index finger from 0 to $90^{\circ}$ and to touch the index finger to the thumb. (B) For the isometric contraction, the volunteer was required to maintain the MP joint of the index finger at $90^{\circ}$ against external extension. 
tasks that involved voluntary contraction. The resting motor threshold was defined as the minimum stimulus intensity that evoked a MEP greater than $50 \mu \mathrm{V}$ in at least five out of ten trials. The active motor threshold was defined as the minimum stimulus intensity that evoked a small MEP $(>100 \mu \mathrm{V})$ in at least five out of ten trials when the volunteer made the FDI muscle contract isometrically, and made the EMG of FDI muscle 5\%MVC with visual EMG feedback.

\section{EXPERIMENTAL PROCEDURE}

The volunteers performed the following experimental tasks in a randomly assigned order (Table 1): (i) shortening contraction with electrical stimulation; (ii) shortening contraction without electrical stimulation; (iii) isometric contraction with electrical stimulation; (iv) isometric contraction without electrical stimulation; (v) electrical stimulation without voluntary contraction. Electrical stimulation included the following two different stimulus conditions: (i) a train of electrical stimuli in which the stimulus duration was set to $5 \mathrm{~s}$; (ii) single electrical pulse just before TMS with the following two different intensities: (i) above motor threshold; (ii) above sensory threshold. A single electrical stimulus just before TMS influences MEPs evoked by TMS in response to the interstimulus interval of a single electrical pulse and a TMS pulse, i.e., short-latency afferent inhibition and afferent facilitation (Mariorenzi et al., 1991; Deletis et al., 1992; Chen et al., 1999; Tokimura etal., 2000; Roy and Gorassini, 2008; Russmann etal., 2009; Devanne etal., 2009), so that a single electrical pulse just before TMS may influence the MEP changes induced by a train of electrical stimuli. Thus, this study investigated not only MEP changes induced by a train of electrical stimuli but also changes induced by a single electrical pulse just before TMS. A total of 14 experimental tasks were performed successively on the same day. For the combination of voluntary hand movement and electrical stimulation, electrical stimulation began to be delivered along with voluntary hand movement and was switched off after $5 \mathrm{~s}$. When a single

Table 1 | Experimental study design.

\section{Voluntary contraction Electrical stimulation (ES)}

Shortening contraction With a train of ES (above motor threshold)

Isometric contraction With a train of ES (above sensory threshold)

With single electrical pulse (above motor threshold)

With single electrical pulse (above sensory threshold)

Without ES

At rest

A train of ES (above motor threshold)

A train of ES (above sensory threshold)

Single electrical pulse (above motor threshold)

Single electrical pulse (above sensory threshold) electrical pulse was combined with voluntary hand movement, electrical stimulation was only delivered just before the TMS trigger.

Transcranial magnetic stimulation measurements were made before and just after the experimental tasks (Figure 2). In TMS measurements before the experimental task, TMS was delivered with $120 \%$ of both the resting and active motor thresholds to measure control MEPs. In TMS measurements after the experimental task, TMS was delivered while the volunteers performed voluntary hand movements. The TMS trigger was applied $60 \mathrm{~ms}$ after the last electrical stimulus pulse to minimize the influence of the electrical pulse on the MEPs and detect the effect of electrical stimulation on the MEPs.

\section{DATA ANALYSIS}

For voluntary contraction with electrical stimulation, the peakto-peak MEP amplitude was measured and expressed relative to the peak-to-peak MEP amplitude observed in the control trial (MEP control ratio). For electrical stimulation without voluntary contraction, the peak-to-peak MEP amplitude was measured. The root mean square (RMS) amplitude of the FDI electromyogram was calculated in the $50 \mathrm{~ms}$ prior to the TMS trigger and expressed relative to the RMS amplitude observed with isometric maximum FDI contraction (RMS maximum ratio). A two-way repeated measured analysis of variance (ANOVA) with factors of muscle contraction (isometric contraction or shortening contraction) and type of electrical stimulation (with a train of electrical stimulation above motor threshold, with a train of electrical stimulation above sensory threshold, with single pulse above motor threshold, with single pulse above sensory threshold or without electrical stimulation) was used to analyze the different effect of electrical stimulation on the corticospinal excitability among the type of muscle contraction

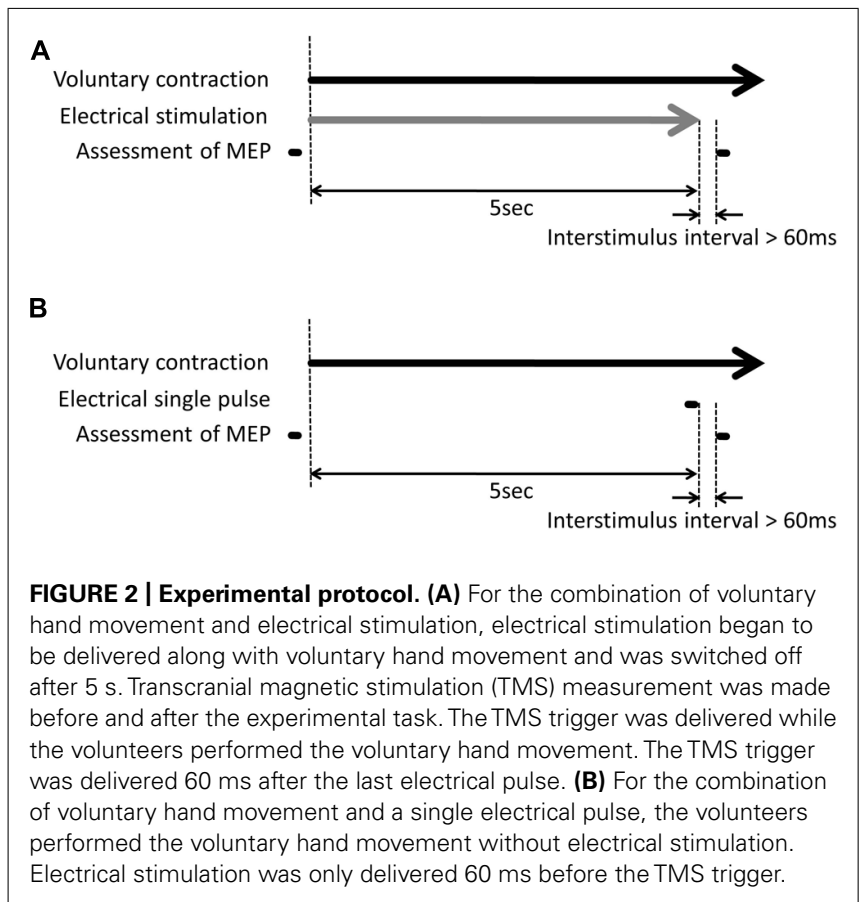


and to analyze the background EMG among each task. For each type of contraction, a repeated-measures ANOVA with the type of contraction as one factor was used to compare the MEP control ratio and the mean RMS maximum ratio across experimental tasks. Post hoc testing was performed using the Tukey multiple comparison. For each type of electrical stimulation, Student's paired $t$-test (two-tailed) was used to compare the MEP control ratio between isometric contraction and shortening contraction. Further, for each stimulus condition of electrical stimulation, a repeated-measures ANOVA with the stimulus condition of electrical stimulation as one factor was used to compare the peak-to-peak MEP amplitude. All statistical analyses were conducted using SPSS 15.0 for Windows. Statistical significance was determined as $p<0.05$ for all comparisons.

\section{RESULTS}

ELECTRICAL STIMULATION-INDUCED MODULATION OF CORTICOSPINAL EXCITABILITY

A two-way repeated-measures ANOVA revealed significant effect of the voluntary hand movement $[F(1.13)=19.638, p=0.001]$ and type of electrical stimulation $[F(4.52)=4.191, p=0.023]$ on the corticospinal excitability. Furthermore, the analysis also revealed significant interaction between voluntary hand movement and the type of electrical stimulation $[F(4.52)=2.956$, $p=0.028]$.

\section{Shortening contraction task}

Figure 3A shows typical MEP waveforms in the FDI muscle from one participant recorded in a shortening FDI contraction performed with and without a single pulse of ulnar nerve

\section{A shortening contraction}

with ES(MT)

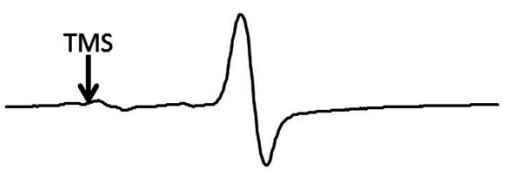

with single pulse(MT)

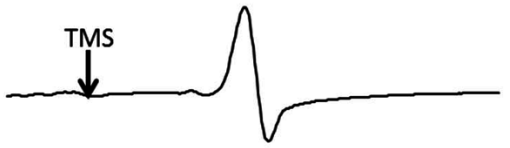

\section{B. isometric contraction}

with ES(MT)

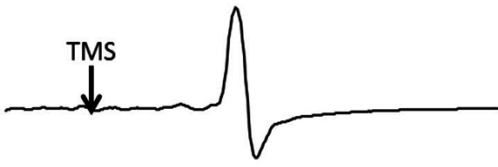

with single pulse(MT)

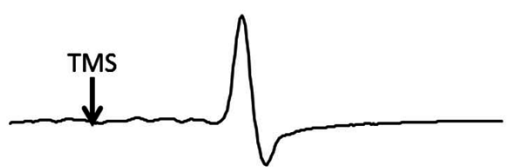

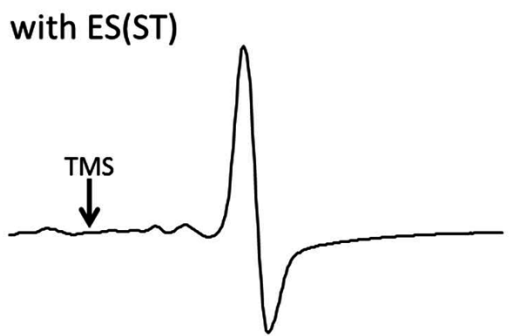

with single pulse(ST)

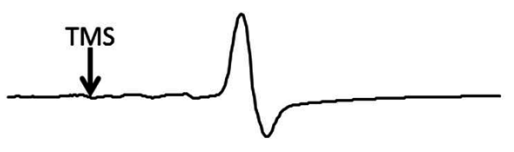

without ES

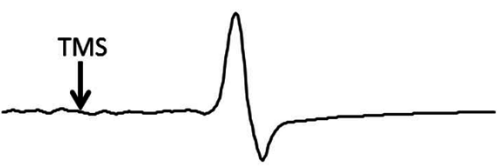

with ES(ST)

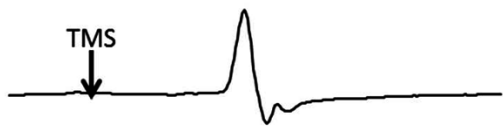

with single pulse(ST)

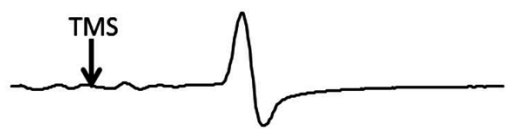

without ES

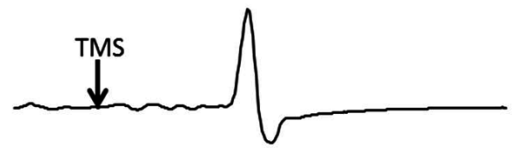

control

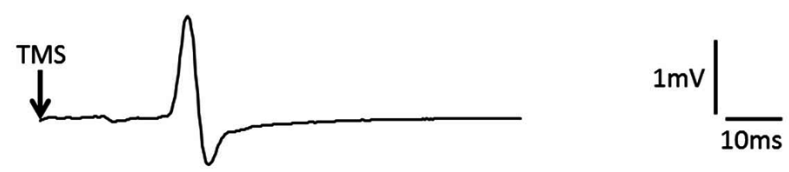

FIGURE 3 | The effect of voluntary contraction with electrical stimulation on the corticospinal excitability in single subject (A) Typical motor-evoked potential (MEP) waveforms recorded in the first dorsal interosseous (FDI) muscle. (A), shortening contraction. (B), isometric contraction. 
stimulation or a train of ulnar nerve stimuli, and Figure 4 shows the pooled data $(n=14)$. A one-way repeated-measures ANOVA revealed a significant effect of the type of electrical stimulation on the MEP control ratio $[F(4.52)=5.002$, $p=0.002]$. Post hoc analysis revealed that the MEP control ratio during shortening contraction was significantly higher with a train of electrical stimulation above sensory threshold than with other types of electrical stimulation $(p<0.05)$. However, the analysis also revealed no significant difference between the MEP control ratio during shortening contraction with electrical stimulation above motor threshold and that without electrical stimulation $(p=0.658)$. These results indicated that a train of low-intensity electrical stimulation was highly effective in facilitating MEPs measured in the FDI during the shortening contraction rather than high-intensity electrical stimulation.

\section{An isometric contraction task}

Figure 3B shows typical MEP waveforms in the FDI muscle from one participant during an isometric contraction performed with and without a single pulse of ulnar nerve stimulation or a train of ulnar nerve stimuli, and Figure 4 shows the pooled data $(n=14)$. A one-way repeated-measures ANOVA revealed that the MEP control ratio was similar across experimental tasks $[F(4.52)=0.600$,

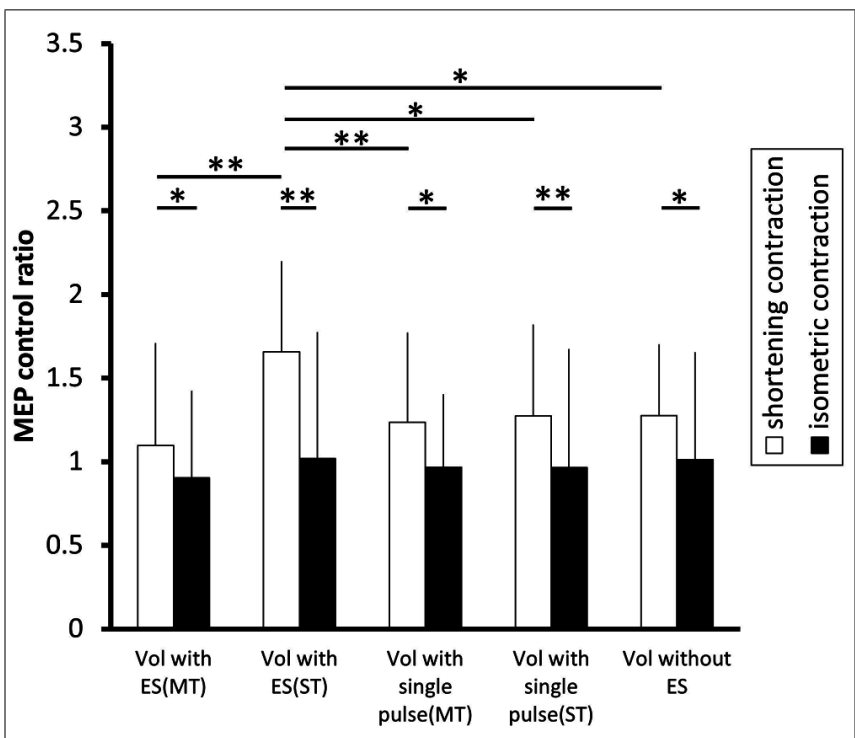

FIGURE 4 | The effect of voluntary contraction with electrical stimulation on the excitability of the corticospinal tract. The changes in the group mean MEP control ratio $(n=14)$ induced by the following experimental tasks: voluntary contraction (vol) with electrical stimulation (ES; above motor threshold, MT), vol with ES (above sensory threshold, ST), vol with a single electrical pulse (MT), vol with a single electrical pulse (ST), and vol without ES. In shortening contraction (white bar), the MEP control ratio was significantly highest when a voluntary shortening contraction was coupled with ES (ST) than when a voluntary shortening contraction was coupled with other types of electrical stimulation (all $p<0.05$ ). In isometric contraction (black bar), the MEP control ratio was similar across experimental tasks $[F(4.52)=0.600, p=0.665]$. Further, the MEP control ratio was significantly higher during shortening contraction than isometric contraction in all type of electrical stimulation (all $p<0.05$ ) ${ }^{*} p<0.05,{ }^{* *} p<0.01$. Error bars indicate standard deviation (SD). $p=0.665]$, indicating that ulnar nerve stimulation did not affect the MEPs measured in the FDI during isometric FDI muscle contraction.

\section{The corticospinal excitability and muscle contraction being performed}

A Student's paired $t$-test revealed that the MEP control ratio was significantly higher during shortening contraction than that during isometric contraction in all types of electrical stimulation $(p<0.05)$, indicating that shortening contraction displayed higher MEPs measured in FDI than isometric contraction regardless of stimulation type.

\section{Electrical stimulation without voluntary contraction}

Figure 5A shows a typical MEP in the FDI from one participant during a train of electrical stimulation of the ulnar nerve without voluntary contraction, and Figure 5B shows the pooled data $(n=14)$. A one-way repeated-measures ANOVA revealed that there was a significant effect of the stimulation condition on the MEP control ratio $[F(4.52)=4.201, p=0.005]$. Post hoc analysis revealed that the MEP amplitude was significantly higher after a train of electrical stimulation above sensory threshold than that after a single pulse above sensory threshold $(p=0.018)$ and that at rest $(p=0.006)$.

\section{RMS ELECTROMYOGRAPHY}

Table 2 shows the average RMS maximum ratio of the FDI electromyography signal in the $50 \mathrm{~ms}$ prior to the TMS trigger. A two-way repeated-measures ANOVA revealed no significant effect of the voluntary hand movement $[F(1.13)=1.004, p=0.335]$ and type of electrical stimulation $[F(4.52)=1.929, p=0.181]$ on the backgroung EMG. Furthermore, the analysis revealed no significant interaction between voluntary hand movement and the type of electrical stimulation $[F(4.52)=1.733, p=0.157]$.

\section{DISCUSSION}

The main finding from these experiments was that a train of electrical stimuli of the ulnar nerve during voluntary hand movement modulated the excitability of the corticospinal projection to the FDI, and that the modulation was dependent on the pattern of muscle contraction. In shortening contraction, low-intensity electrical stimulation displayed increased corticospinal excitability compared with high-intensity stimulation. In isometric contraction, a train of electrical stimulation did not affect the excitability of the corticospinal tract. On the other hand, in resting condition, low-intensity electrical stimulation was effective for increasing the corticospinal excitability.

\section{THE EFFECT OF SHORTENING CONTRACTION WITH ELECTRICAL STIMULATION ON THE CORTICOSPINAL EXCITABILITY}

During the shortening contraction task, low-intensity electrical nerve stimulation increased the excitability of the corticospinal tract, but high-intensity electrical nerve stimulation did not. This is consistent with the results found in our previous study, in which the effect of electrical stimulation on corticospinal excitability was dependent on stimulus intensity (Saito et al., 2013).

The additive effect of increased corticospinal excitability by shortening contraction, plus increased excitability by electrical 

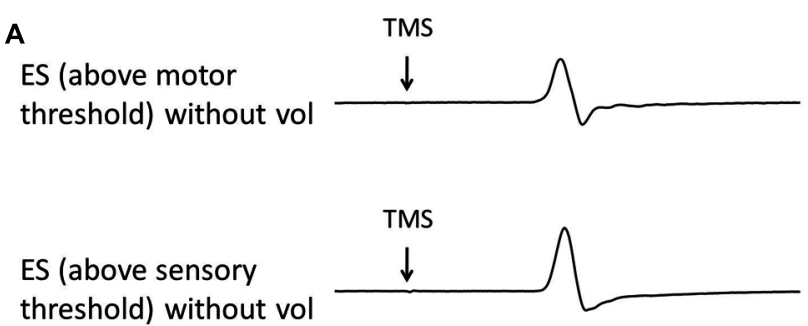
threshold) without vol

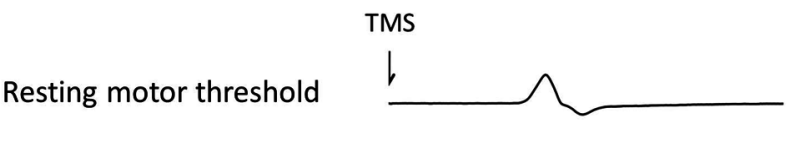

Single electrical

pulse (above motor

threshold) without vol

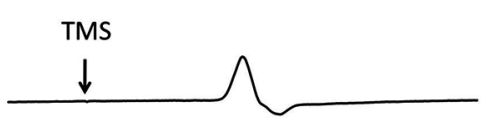

Single electrical

pulse (above sensory

threshold) without vol

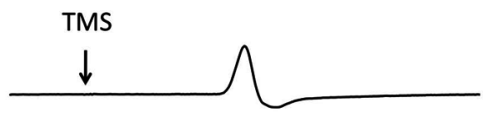

B

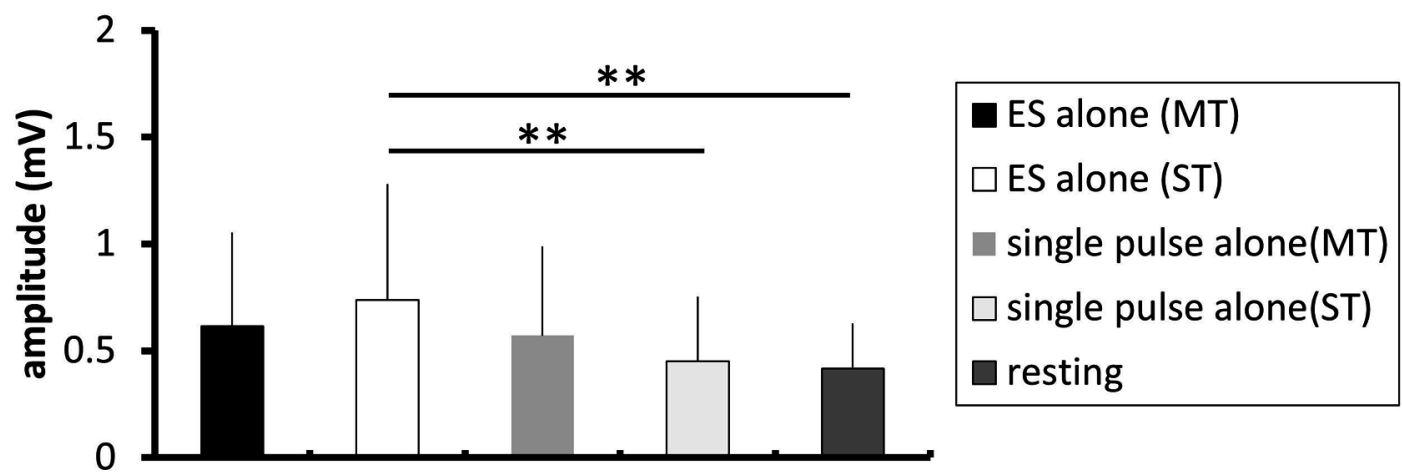

FIGURE 5 | The effect of electrical stimulation without voluntary contraction on the excitability of the corticospinal tract. (A) Typical averaged motor-evoked potential (MEP) waveforms recorded in the FDI muscle. (B) The changes in the group mean MEP control ratio $(n=8)$ induced by the following experimental tasks: electrical stimulation (ES; above motor threshold, MT) without voluntary contraction (vol), ES (above sensory threshold, ST) without vol, single electrical pulse (MT) without vol, and single electrical pulse (ST) without vol, and a control trial using the resting motor threshold as the TMS intensity. The MEP amplitude was significantly higher after a train of electrical stimulation above sensory threshold than after single pulse above sensory threshold ( $p=0.018)$ and at rest $(p=0.006)$.

Table 2 | Background activity of the first dorsal interosseous (FDI) muscle during each task.

The type of voluntary contraction

\begin{tabular}{llc} 
& \multicolumn{1}{c}{ The type of voluntary contraction } \\
\cline { 2 - 3 } & Shortening contraction & Isometric contraction \\
\hline Vol with ES (above motor threshold) & $0.16 \pm 0.04$ & $0.16 \pm 0.03$ \\
Vol with ES (above sensory threshold) & $0.14 \pm 0.05$ & $0.13 \pm 0.04$ \\
Vol with single electrical pulse (above motor threshold) & $0.16 \pm 0.08$ & $0.14 \pm 0.08$ \\
Vol with single electrical pulse (above sensory threshold) & $0.14 \pm 0.04$ & $0.14 \pm 0.04$ \\
Vol without ES & $0.13 \pm 0.06$ & $0.13 \pm 0.05$ \\
\hline
\end{tabular}

Average $\pm S D$

stimulation, may explain the additional increase in corticospinal excitability during shortening contraction combined with lowintensity electrical stimulation. A previous study reported that corticospinal excitability is highly increased during a shortening contraction (Kasai etal., 1997). Furthermore, our study also showed that low-intensity electrical stimulation displayed a significantly increased corticospinal excitability when the stimulated muscle was at rest. This is consistent with the observations of previous studies showing that electrical stimulation increases corticospinal excitability (Ridding et al., 2000, 2001; Kaelin-Lang et al., 2002; Khaslavskaia et al., 2002; Mckay et al., 2002; Charlton et al., 2003; Knash et al., 2003; Tinazzi et al., 2005; Mang et al., 2010, 2011; Chipchase et al., 2011a,b; Golaszewski etal., 2012; Andrews et al., 2013). The combination of electrical stimulation with voluntary contraction yielded the greatest increase in corticospinal excitability (Khaslavskaia and Sinkjaer, 2005), suggesting 
these inputs may induce activation of multiple pyramidal cells, leading to a state of subliminal fringe. Consequently, we believe the TMS pulse was able to activate more pyramidal cells during a shortening contraction with low-intensity electrical stimulation.

In contrast, the additive effect might be specific for lowintensity electrical stimulation with shortening contraction. This study showed that high-intensity electrical stimulation was not effective for modulating the corticospinal excitability during shortening contraction. Considering that high-intensity electrical stimulation did not affect corticospinal excitability when the stimulated muscle was at rest, afferent input by high-intensity electrical stimulation might be insufficient to modulate corticospinal excitability. Thus, the additive effect might not be induced, even when shortening contraction is combined with high-intensity electrical stimulation.

\section{THE EFFECT OF ISOMETRIC CONTRACTION WITH ULNAR NERVE STIMULATION ON THE CORTICOSPINAL EXCITABILITY}

Electrical stimulation of the ulnar nerve did not affect the corticospinal excitability when the volunteer was maintaining a constant index finger MP joint angle while exerting a constant torque against a rigid restraint. This is not consistent with the observation that low-intensity electrical stimulation but not high-intensity electrical stimulation is effective in increasing corticospinal excitability. These results indicated that isometric contraction might result in a loss of the lowintensity electrical stimulation-induced effect on the corticospinal excitability.

This discrepancy might be due to the movement-related gating of the sensory input. Previous studies have demonstrated gating of sensory input during voluntary contraction (Angel and Malenka, 1982; Blakemore et al., 1998; Bays et al., 2005), indicating attenuation of afferent input during the contraction. Thus, in this study, afferent input by electrical stimulation might be reduced by sensory gating during isometric contraction. Furthermore, the duration of electrical nerve stimulation in the present study (5s), was shorter than that used in previous studies, where electrical stimulation increased the corticospinal excitability (Ridding et al., 2000, 2001; Mang et al., 2011; Golaszewski et al., 2012; Léonard et al., 2013). Thus, the combined use of isometric contraction and electrical stimulation may not have been sufficient to increase corticospinal excitability.

\section{THE CORTICOSPINAL EXCITABILITY AND MUSCLE CONTRACTION BEING PERFORMED}

The corticospinal excitability was significantly higher during shortening contraction than that during isometric contraction regardless of the intensity and type of electrical stimulation.

The different level of movement-related sensory gating between the muscle contractions being performed is believed to be responsible for this result. Previous studies have demonstrated gating of sensory input during voluntary contraction (Angel and Malenka, 1982; Blakemore etal., 1998; Bays et al., 2005). Another study showed that a shortening contraction increased the SEP over that of isometric contraction (Lee and White, 1974). These results indicate the level of somatosensory processing that induces attenuation of somatosensory input is stronger during isometric contraction than during shortening contraction. Thus, in this study, the level of sensory gating during isometric contraction with electrical stimulation is likely to be stronger than that during shortening contraction with electrical stimulation.

\section{THE EFFECT OF ULNAR NERVE STIMULATION WITHOUT VOLUNTARY CONTRACTION ON THE CORTICOSPINAL EXCITABILITY}

Low-intensity electrical stimulation was effective in increasing the corticospinal excitability, but high-intensity electrical stimulation was not effective. These results indicated that the effect of electrical stimulation on corticospinal excitability was specific for the stimulation intensity. This is not consistent with the observation from the previous studie, where the increase in the corticospinal excitability elicited by electrical stimulation was reported to be dependent on stimulus intensity (Khaslavskaia et al., 2002).

This discrepancy might be due to sensory gating induced by electrical muscle contraction. A previous study reported that gating of sensory input was present during passive movement as well as during voluntary movement (Jones et al., 1989). High-intensity electrical stimulation used in this study caused muscle contraction without voluntary effort and consequently might have induced the gating of sensory input as well as voluntary contraction. Along with the short duration of electrical nerve stimulation in the present study (5 s), high-intensity electrical stimulation that has been used here might be insufficient to increase the corticospinal excitability.

\section{METHODOLOGICAL CONSIDERATIONS}

An increase or decrease in the excitability of spinal interneurons may be involved in an increase in the excitability of the corticospinal tract to the FDI muscle. Christova et al. (2010) reported that muscle vibration that externally stimulates the muscle spindle does not regulate the excitability of the spinal cord during a voluntary contraction. Furthermore, electrical stimulation does not affect the excitability of the spinal cord (Thompson et al., 2006). Thus, electrical stimulation-induced modulation of corticospinal excitability during voluntary contraction may be predominantly caused by a change in the excitability of the primary motor cortex. Further examination of the mechanism underlying the changes in corticospinal excitability induced by the combination of voluntary contraction and electrical stimulation is necessary.

\section{CONCLUSION}

Our results suggest that electrical nerve stimulation-induced modulation of corticospinal excitability is dependent on the type of voluntary muscle contraction being performed. This indicates that the intensity of electrical stimulation should be set according to the hand motor task being performed when a combination of voluntary contraction and electrical stimulation is utilized for rehabilitation of patients with central nervous system lesions.

\section{AUTHOR CONTRIBUTIONS}

Kei Saito, Kenichi Sugawara, and Hideaki Onishi. Performed the experiments: Kei Saito, Shota Miyaguchi, Takuya Matsumoto, and Hideaki Onishi. Analyzed the data: Kei Saito, Hideaki Onishi, Hikari Kirimoto, Hiroyuki Tamaki, and Kenichi Sugawara. Wrote 
the paper: Kei Saito, Hideaki Onishi, Kenichi Sugawara, Hikari Kirimoto, and Hiroyuki Tamaki.

\section{ACKNOWLEDGMENTS}

We thank all the volunteers for their time and patience during testing. This study was supported by a Grant-in-Aid for Exploratory Research from Niigata University of Health and Welfare.

\section{REFERENCES}

Andrews, R. K., Schabrun, S. M., Ridding, M. C., Galea, M. P., Hodges, P. W. and Chipchase, L. S. (2013). The effect of electrical stimulation on corticospinal excitability is dependent on application duration: a same subject pre-post test design. J. Neuroeng. Rehabil. 10, 51. doi: 10.1186/1743-0003-10-51

Angel, R. W., and Malenka, R. C. (1982). Velocity-dependent suppression of cutaneous sensitivity during movement. Exp. Neurol. 77, 266-274. doi: 10.1016/0014-4886(82)90244-8

Barsi, G. I., Popovic, D. B., Tarkka, I. M., Sinkjaer, T., and Grey, M. J. (2008). Cortical excitability changes following grasping exercise augmented with electrical stimulation. Exp. Brain Res. 191, 57-66. doi: 10.1007/s00221-0081495-5

Bays, P. M., Wolpert, D. M., and Flanagan, J. R. (2005). Perception of the consequences of self-action is temporally tuned and event driven. Curr. Biol. 15, 1125-1128. doi: 10.1016/j.cub.2005.05.023

Bhatt, E., Nagpal, A., Greer, K. H., Grunewald, T. K., Steele, J. L., Wiemiller, J. W., et al. (2007). Effect of finger tracking combined with electrical stimulation on brain reorganization and hand function in subjects with stroke. Exp. Brain Res. 182, 435-447. doi: 10.1007/s00221-007-1001-5

Blakemore, S. J., Wolpert, D. M., and Frith, C. D. (1998). Central cancellation of self-produced tickle sensation. Nat. Neurosci. 1, 635-640. doi: 10.1038/2870

Bolton, D. A., Cauraugh, J. H., and Hausenblas, H. A. (2004) Electromyogram-triggered neuromuscular stimulation and stroke motor recovery of arm/hand functions: a meta-analysis. J. Neurol. Sci. 223, 121-127. doi 10.1016/j.jns.2004.05.005

Charlton, C. S., Ridding, M. C., Thompson, P. D., and Miles, T. S. (2003). Prolonged peripheral nerve stimulation induces persistent changes in excitability of human motor cortex. J. Neurol. Sci. 208, 79-85. doi: 10.1016/S0022-510X(02)00443-4

Chen, R., Corwell, B., and Hallett, M. (1999). Modulation of motor cortex excitability by median nerve and digit stimulation. Exp. Brain Res. 129, 77-86. doi: $10.1007 / \mathrm{s} 002210050938$

Chipchase, L. S., Schabrun, S. M., and Hodges, P. W. (2011a). Corticospina excitability is dependent on the parameters of peripheral electric stimulation: a preliminary study. Arch. Phys. Med. Rehabil. 92, 1423-1430. doi 10.1016/j.apmr.2011.01.011

Chipchase, L. S., Schabrun, S. M., and Hodges, P. W. (2011b). Peripheral electrical stimulation to induce cortical plasticity: a systematic review of stimulus parameters. Clin. Neurophysiol. 122, 456-463. doi: 10.1016/j.clinph.2010.07.025

Christova, M., Rafolt, D., Mayr, W., Wilfling, B., and Gallasch, E. (2010). Vibration stimulation during non-fatiguing tonic contraction induces outlasting neuroplastic effects. J. Electromyogr. Kinesiol. 20, 627-635. doi: 10.1016/j.jelekin.2010.03.001

Chye, L., Nosaka, K., Murray, L., Edwards, D., and Thickbroom, G. (2010). Corticomotor excitability of wrist flexor and extensor muscles during active and passive movement. Hum. Mov. Sci. 29, 494-501. doi: 10.1016/j.humov.2010.03.003

de Kroon, J. R., Ijzerman, M. J., Chae, J., Lankhorst, G. J., and Zilvold, G. (2005). Relation between stimulation characteristics and clinical outcome in studies using electrical stimulation to improve motor control of the upper extremity in stroke. J. Rehabil. Med. 37, 65-74. doi: 10.1080/16501970410024190

Deletis, V., Schild, J. H., Berić, A., and Dimitrijević, M. R. (1992). Facilitation of motor evoked potentials by somatosensory afferent stimulation. Electroencephalogr. Clin. Neurophysiol. 85, 302-310. doi: 10.1016/0168-5597(92)90106-L

Devanne, H., Degardin, A., Tyvaert, L., Bocquillon, P., Houdayer, E., Manceaux, A., et al. (2009). Afferent-induced facilitation of primary motor cortex excitability in the region controlling hand muscles in humans. Eur. J. Neurosci. 30, 439-448. doi: $10.1111 / j .1460-9568.2009 .06815 . x$

Fujiwara, T., Kasashima, Y., Honaga, K., Muraoka, Y., Tsuji, T., Osu, R., et al. (2009). Motor improvement and corticospinal modulation induced by hybrid assistive neuromuscular dynamic stimulation (HANDS) therapy in patients with chronic stroke. Neurorehabil. Neural Repair 23, 125-132. doi: 10.1177/1545968308321777
Golaszewski, S. M., Bergmann, J., Christova, M., Kunz, A. B., Kronbichler, M., Rafolt, D., et al. (2012). Modulation of motor cortex excitability by different levels of whole-hand afferent electrical stimulation. Clin. Neurophysiol. 123, 193-199. doi: 10.1016/j.clinph.2011.06.010

Hara, Y., Obayashi, S., Tsujiuchi, K., and Muraoka, Y. (2013). The effects of electromyography-controlled functional electrical stimulation on upper extremity function and cortical perfusion in stroke patients. Clin. Neurophysiol. 124, 2008-2015. doi: 10.1016/j.clinph.2013.03.030

Jones, S. J., Halonen, J. P., and Shawkat, F. (1989). Centrifugal and centripetal mechanisms involved in the 'gating' of cortical SEPs during movement. Electroencephalogr. Clin. Neurophysiol. 74, 36-45. doi: 10.1016/0168-5597(89) 90049-X

Kaelin-Lang, A., Luft, A. R., Sawaki, L., Burstein, A. H., Sohn, Y. H., and Cohen, L. G. (2002). Modulation of human corticomotor excitability by somatosensory input. J. Physiol. 540, 623-633. doi: 10.1113/jphysiol.2001.012801

Kasai, T., Toyoda, Y., and Yahagi, S. (1997). Motor evoked potentials (MEPs) and H-reflexes are not equally sensitive to voluntary motor commands. Brain Res. 764, 273-276. doi: 10.1016/S0006-8993(97)00598-2

Khaslavskaia, S., Ladouceur, M., and Sinkjaer, T. (2002). Increase in tibialis anterior motor cortex excitability following repetitive electrical stimulation of the common peroneal nerve. Exp. Brain Res. 145, 309-315. doi: 10.1007/s00221-0021094-9

Khaslavskaia, S., and Sinkjaer, T. (2005). Motor cortex excitability following repetitive electrical stimulation of the common peroneal nerve depends on the voluntary drive. Exp. Brain Res. 162, 497-502. doi: 10.1007/s00221-004-2153-1

Kido Thompson, A., and Stein, R. B. (2004). Short-term effects of functional electrical stimulation on motor-evoked potentials in ankle flexor and extensor muscles. Exp. Brain Res. 159, 491-500. doi: 10.1007/s00221-004-1972-4

Kimberley, T. J., Lewis, S. M., Auerbach, E. J., Dorsey, L. L., Lojovich, J. M., and Carey, J. R. (2004). Electrical stimulation driving functional improvements and cortical changes in subjects with stroke. Exp. Brain Res. 154, 450-460. doi: 10.1007/s00221-003-1695-y

Knash, M. E., Kido, A., Gorassini, M., Chan, K. M., and Stein, R. B. (2003). Electrical stimulation of the human common peroneal nerve elicits lasting facilitation of cortical motor-evoked potentials. Exp. Brain Res. 153, 366-377. doi: 10.1007/s00221-003-1628-9

Lee, R. G., and White, D. G. (1974). Modification of the human somatosensory evoked response during voluntary movement. Electroencephalogr. Clin. Neurophysiol. 36, 53-62. doi: 10.1016/0013-4694(74)90136-9

Léonard, G., Mercier, C., and Tremblay, L. E. (2013). Effect of repetitive afferent electrical stimulation of the lower limb on corticomotor excitability and implications for rehabilitation. J. Clin. Neurosci. 20, 435-439. doi: 10.1016/j.jocn.201 2.02.049

Mang, C. S., Clair, J. M., and Collins, D. F. (2011). Neuromuscular electrical stimulation has a global effect on corticospinal excitability for leg muscles and a focused effect for hand muscles. Exp. Brain Res. 209, 355-363. doi: 10.1007/s00221-011-2556-8

Mang, C. S., Lagerquist, O., and Collins, D. F. (2010). Changes in corticospinal excitability evoked by common peroneal nerve stimulation depend on stimulation frequency. Exp. Brain Res. 203, 11-20. doi: 10.1007/s00221-010-2202-x

Mariorenzi, R., Zarola, F., Caramia, M. D., Paradiso, C., and Rossini, P. M. (1991). Non-invasive evaluation of central motor tract excitability changes following peripheral nerve stimulation in healthy humans. Electroencephalogr. Clin. Neurophysiol. 81, 90-101. doi: 10.1016/0168-5597(91)90002-F

McDonnell, M. N., Hillier, S. L., Miles, T. S., Thompson, P. D., and Ridding, M. C. (2007). Influence of combined afferent stimulation and task-specific training following stroke: a pilot randomized controlled trial. Neurorehabil. Neural Repair 21, 435-443. doi: 10.1177/1545968307300437

Mckay, D., Brooker, R., Giacomin, P., Ridding, M., and Miles, T. (2002). Time course of induction of increased human motor cortex excitability by nerve stimulation. Neuroreport 13, 1271-1273. doi: 10.1097/00001756-200207190-00011

Ridding, M. C., Brouwer, B., Miles, T. S., Pitcher, J. B., and Thompson, P. D. (2000). Changes in muscle responses to stimulation of the motor cortex induced by peripheral nerve stimulation in human subjects. Exp. Brain Res. 131, 135-143. doi: $10.1007 / \mathrm{s} 002219900269$

Ridding, M. C., Mckay, D. R., Thompson, P. D., and Miles, T. S. (2001). Changes in corticomotor representations induced by prolonged peripheral nerve stimulation in humans. Clin. Neurophysiol. 112, 1461-1469. doi: 10.1016/S13882457(01)00592-2 
Roy, F. D., and Gorassini, M. A. (2008). Peripheral sensory activation of cortical circuits in the leg motor cortex of man. J. Physiol. 586, 4091-4105. doi: 10.1113/jphysiol.2008.153726

Russmann, H., Lamy, J. C., Shamim, E. A., Meunier, S., and Hallett, M. (2009). Associative plasticity in intracortical inhibitory circuits in human motor cortex. Clin. Neurophysiol. 120, 1204-1212. doi: 10.1016/j.clinph.2009.04.005

Saito, K., Yamaguchi, T., Yoshida, N., Tanabe, S., Kondo, K., and Sugawara, K. (2013). Combined effect of motor imagery and peripheral nerve electrical stimulation on the motor cortex. Exp. Brain Res. 227, 333-342. doi: 10.1007/s00221-013-3513-5

Seki, K., and Fetz, E. E. (2012). Gating of sensory input at spinal and cortical levels during preparation and execution of voluntary movement. J. Neurosci. 32, 890-902. doi: 10.1523/JNEUROSCI.4958-11.2012

Sugawara, K., Yamaguchi, T., Tanabe, S., Suzuki, T., Saito, K., and Higashi, T. (2013). Time-dependent changes in motor cortical excitability by electrical stimulation combined with voluntary drive. Neuroreport 25, 404-409. doi:10.1097/WNR.0000000000000108

Tarkka, I. M., Pitkänen, K., Popovic, D. B., Vanninen, R., and Könönen, M. (2011). Functional electrical therapy for hemiparesis alleviates disability and enhances neuroplasticity. Tohoku J. Exp. Med. 225, 71-76. doi: 10.1620/tjem.225.71

Thompson, A. K., Doran, B., and Stein, R. B. (2006). Short-term effects of functional electrical stimulation on spinal excitatory and inhibitory reflexes in ankle extensor and flexor muscles. Exp. Brain Res. 170, 216-226. doi: 10.1007/s00221-005-0203-y

Tinazzi, M., Zarattini, S., Valeriani, M., Romito, S., Farina, S., Moretto, G., et al. (2005). Long-lasting modulation of human motor cortex following prolonged transcutaneous electrical nerve stimulation (TENS) of forearm muscles: evidence of reciprocal inhibition and facilitation. Exp. Brain Res. 161, 457-464. doi: 10.1007/s00221-004-2091-y

Tokimura, H., Di Lazzaro, V., Tokimura, Y., Oliviero, A., Profice, P., Insola, A., et al. (2000). Short latency inhibition of human hand motor cortex by somatosensory input from the hand. J. Physiol. 523, 503-513. doi: 10.1111/j.1469-7793.2000.t011-00503.x

Conflict of Interest Statement: The authors declare that the research was conducted in the absence of any commercial or financial relationships that could be construed as a potential conflict of interest.

Received: 18 July 2014; accepted: 30 September 2014; published online: 16 October 2014.

Citation: Saito K, Sugawara K, Miyaguchi S, Matsumoto T, Kirimoto H, Tamaki H and Onishi $H$ (2014) The modulatory effect of electrical stimulation on the excitability of the corticospinal tract varies according to the type of muscle contraction being performed. Front. Hum. Neurosci. 8:835. doi: 10.3389/fnhum.2014.00835

This article was submitted to the journal Frontiers in Human Neuroscience.

Copyright (C) 2014 Saito, Sugawara, Miyaguchi, Matsumoto, Kirimoto, Tamaki and Onishi. This is an open-access article distributed under the terms of the Creative Commons Attribution License (CC BY). The use, distribution or reproduction in other forums is permitted, provided the original author(s) or licensor are credited and that the original publication in this journal is cited, in accordance with accepted academic practice. No use, distribution or reproduction is permitted which does not comply with these terms. 